\title{
TRATAMENTOS ALTERNATIVOS DO EFLUENTE DE UMA INDÚSTRIA DE CELULOSE BRANQUEADA E PAPEL
}

\section{ALTERNATIVES OF AN EFFLUENT FROM AN BLECHEAD PULP AND TREATMENTS PAPER INDUSTRY}

\author{
Dorotéia Maria Martins Flores ${ }^{1}$ Sonia Maria Bitencourt Frizzo ${ }^{2}$ \\ Celso Edmundo Bochetti Foelkel ${ }^{3}$
}

\begin{abstract}
RESUMO
Este trabalho procurou avaliar a eficiência de tratamento floculante com sulfato de alumínio ou cloreto férrico para o efluente bruto neutralizado de uma indústria de celulose e papel. A filtração do efluente, removendo fibras, finos, macromoléculas e flocos naturais, permitiu expressivas reduções da cor aparente, cor real e DQO do mesmo. Enquanto o sulfato de alumínio mostrou excelentes resultados, o cloreto férrico não teve bom desempenho. Deve-se otimizar as concentrações, as formas de agitação e a decantação dos flocos. Para as amostras testadas, na primeira seqüência de testes, concentrações com cerca de 2500 ppm de sulfato de alumínio (com 14 moléculas de água de hidratação) mostraram ótimos resultados para redução de cor aparente, cor real, DQO e cloretos. Conforme o previsto, na segunda seqüência de testes as concentrações de $\left[\mathrm{Al}_{2}\left(\mathrm{SO}_{4}\right)_{3}\right.$. $\left.14 \mathrm{H}_{2} \mathrm{O}\right]$ diminuíram para todos os índices físico-químicos testados. A melhor concentração para $\mathrm{pH}$, cor aparente e cor real foi de $1000 \mathrm{ppm}$ filtrado, enquanto que para cloretos foi de 2000 ppm não filtrado, com o tempo de agitação de 1 minuto com alta velocidade e 15 minutos à baixa velocidade, com exceção da cor real que foi de 10 minutos à baixa velocidade. Como recomendação, sugere-se avaliar uma etapa seqüencial de filtração após clarifloculação/decantação. Com isso, o desempenho de um tratamento físico-químico de efluentes desse tipo será eficiente, apesar do custo envolvido na aquisição do insumo sulfato de alumínio.
\end{abstract}

Palavras-chave: Efluente, indústria de celulose e papel, clarifloculação.

1. Enga . Florestal, M.Sc. pelo Programa de Pós-graduação em Engenharia Florestal/CCR/UFSM. CEP: 97105-900. Santa Maria. RS.

2. Enga ${ }^{a}$ Florestal, M.Sc., Prof ${ }^{\mathrm{a}}$. Adjunta do Departamento de Química/CCNE/UFS. CEP: 97105900. Santa Maria. RS.

3. Eng. Agrônomo, Dr. hc., RIOCELL S.A., Prof. do Programa de Pós-graduação em Engenharia Florestal/CCR/UFSM. CEP: 92500-000. Guaíba. RS. 


\begin{abstract}
This paper tried to evaluate the efficiency of the flakelet treatment with aluminum sulphate or ferric chloride for the neutralized brute effluent of a pulp and paper industry. The effluent filtration, removing fibers, fines macromolecules and natural flakes, permited expressive reductions of the apparent color, real color and COD of the same. While the aluminum sulphate showed excellent results, the ferric chloride didn't have good performance. It should optimize the concentrations the stirring forms and the flakes decantation. To the samples tested in the first sequence of tests, concentrations around 2,500 ppm of aluminum sulphate (with 14 water molecules of hydration) they showed excelent results to the reduction of the apparent and real color, COD and clorides. As it was foreseen, in the second sequence of tests, the concentrations of $\left[\mathrm{Al}_{2}\left(\mathrm{SO}_{4}\right)_{3} \cdot 14 \mathrm{H}_{2} \mathrm{O}\right]$ decresead to all the physicochemical index testad. The best concentration to $\mathrm{pH}$, apparent color and real color was of $1,000 \mathrm{ppm}$ filtrated, while the chorides it was $2000 \mathrm{ppm}$ not filtrated, with one minute [1] in highspeed and fifteen [15] minutes in low speed, stirring time except the real color that was ten [10] minutes in low speed. A recommendation, is suggested to evaluate a sequencial phase of filtration after (clarifloculation/decantation). With this, the performance of a physicochemical treatment of the effluents of this type will be efficient, in spite of the cost involved in the acquisition of aluminum sulphate supply.
\end{abstract}

Key words: Effluent, pulp and paper industry, clarifloculation.

\title{
INTRODUÇÃO
}

O tratamento de efluentes industriais na área de papel e celulose é de grande importância, devido a necessidade de diminuir-se a quantidade de contaminantes e pela possível poluição dos corpos receptores d'água (SUPERSULFATO INDÚSTRIA QUÍMICA, 1988).

As indústrias de celulose e papel geram grande quantidade de resíduos sólidos, efluentes hídricos e emissões gasosas, que podem ser lançadas ao meio ambiente. Essas indústrias consomem grande volume de água que após o uso são devolvidos aos corpos receptores, sob a forma de efluentes (CINQUE et al.,1996).

O controle de efluentes industriais, baseia-se em análises químicas dos despejos, e tem como finalidade reduzir a contaminação nos recursos hídricos (BERTOLLETI, 1990). Esses despejos podem modificar a qualidade da água, a flora e a fauna causando poluição ambiental (FROEHLICH, 1987).

$\mathrm{Na}$ indústria de celulose e papel a redução de descargas de contaminantes pode ser feita ou pela mudança no processo de branqueamento da polpa ou pela alteração no tratamento do efluente (ERIKSSON, 1991), ou pelo sistema de monitoramento contínuo de efluentes, o que forneceria dados para o controle dos efluentes e operações dos sistemas de tratamentos, com possibilidade de prevenção de impactos ambientais (LANDIM \& OLIVEIRA, 1995).

As indústrias estão adotando tecnologias de processos e de controle ambiental que permitem

Ciência Florestal, v.8, n.1, 1998 
garantir a menor geração de efluentes hídricos, aéreos e resíduos sólidos, permitindo assim o controle e buscando a minimização dos impactos ambientais (HOUK, 1992).

A presença de compostos tóxicos nos efluentes tem sido motivo de grande preocupação e de estudos, não só em países desenvolvidos como também no Brasil (BORGES et al., 1994).

O presente trabalho, buscou avaliar o efluente bruto neutralizado de uma indústria de celulose e papel com relação aos índices físico-químicos, aplicando tratamentos e procurando alternativas para melhoria de sua qualidade. A indústria RIOCELL S.A. possui um eficiente sistema de tratamento de efluentes, consistindo de um tratamento primário (gradeamento, decantação e redução de temperatura), uma etapa secundária biológica por lodo ativado/aeróbico e um tratamento terciário (floculação por sulfato de alumínio). Quando o efluente alcança o tratamento terciário já ocorreu uma expressiva redução da Demanda Química de Oxigênio (DQO) e Demanda Biológica de Oxigênio (DBO) do efluente, mas a cor ainda persiste, dai a razão para a floculação.

Finalmente objetivou-se estudar apenas uma das etapas de tratamento ao efluente bruto neutralizado (amostra colhida sem nenhum tratamento), para que, no caso de perda ou estrago, por parte da indústria, do tratamento biológico, esta pudesse apenas com a clarifloculação/decantação, obter bons resultados só com o uso de sulfato de alumínio ou de cloreto férrico.

\section{MATERIAL E MÉTODOS}

\section{Amostragem}

As amostras foram coletadas do efluente bruto neutralizado, da indústria Riocell S.A., sem que sofressem nenhum tipo de tratamento. Estas amostras foram armazenadas em recipientes descontaminados e conservadas em câmara fria a mais ou menos $2^{\circ} \mathrm{C}$ (APHA AWWA, 1985 e DIN, 1985).

\section{Desenvolvimento do trabalho}

Foram colhidas duas amostras do efluente para a realização de duas seqüências de testes.

Em ambos os casos o efluente era separado em duas porções onde uma foi filtrada, para remoção dos sólidos suspensos, sendo a outra não filtrada. O trabalho permitiu, num primeiro momento, avaliar a participação dos sólidos suspensos na qualidade físico-química do efluente. Foram avaliados: $\mathrm{pH}$, cor aparente, cor real, DQO (demanda química de oxigênio) e cloretos.

A seguir, tanto a amostra do efluente não filtrado como a amostra do efluente filtrado foi submetida à clarifloculação. Foram usados dois agentes floculantes sulfato de alumínio $\left[\mathrm{Al}\left(\mathrm{SO}_{4}\right)_{3}\right.$. $14 \mathrm{H}_{2} \mathrm{O}$ ] e cloreto férrico $\left[\mathrm{FeCl}_{3} .6 \mathrm{H}_{2} \mathrm{O}\right.$ ] (FRIZZO et al., 1996). O pH inicial era ajustado para permitir melhor efeito da floculação: para o sulfato de alumínio era fixado em 4,7 e para cloreto férrico 4,0. As concentrações aplicadas de $\left[\mathrm{Al}\left(\mathrm{SO}_{4}\right)_{3} .14 \mathrm{H}_{2} \mathrm{O}\right]$ foram de 2.500, 2.800 e $3.000 \mathrm{ppm}$ na primeira seqüência de testes. Para comparação, foram aplicados 3.000, 3.500 e 4.000 ppm de $\left[\mathrm{FeCl}_{3}\right.$. $\left.6 \mathrm{H}_{2} \mathrm{O}\right]$ 
E na segunda seqüência de testes, já com outra amostra de efluente, as concentrações de sulfato de alumínio foram 1.000, 1.500 e $2.000 \mathrm{ppm}$. Não foi utilizado cloreto férrico nesta sequiência de testes. A razão, para esta segunda sequiência foi otimizar a forma de aplicação do sulfato de alumínio. Ao mesmo tempo procurou-se avaliar o efeito da redução do tempo de agitação na fase lenta de 15 para 10 minutos.

Em todos os casos, as amostras do efluente (não filtrado e filtrado) foram estudadas de acordo com as seguintes etapas: verificação inicial das características físico-químicas ( $\mathrm{pH}$, cor aparente, cor real, DQO, cloretos); aplicação do tratamento floculação/decantação; verificação da qualidade do efluente final (sobrenadante).

Na primeira sequiência de testes a agitação após a adição do floculante foi feita em duas etapas:

- fase rápida: um minuto a $300 \mathrm{rpm}$

- fase lenta: quinze minutos a $50 \mathrm{rpm}$

$\mathrm{Na}$ segunda seqüência de testes, procurou-se otimizar a fase lenta de agitação, trabalhando com dez e quinze minutos na fase lenta $(50 \mathrm{rpm})$, mantida a fase rápida $(300 \mathrm{rpm})$ similar para os dois casos. Com o auxílio do teste de jarro, o programa de agitação foi realizado através de um agitador mecânico, com capacidade de um litro, possuindo hélice de vidro. Após a aplicação dos tratamentos deixaram-se as amostras em repouso por 30 minutos para decantar. Posteriormente, foram realizadas no efluente tratado, filtrado e não filtrado, as determinações de $(\mathrm{pH}$, cores aparente e real, DQO e cloretos) para a verificação ou não de sua eficiência.

A verificação das condições físico-químicas seguiu a seguinte metodologia:

- $\mathrm{pH}$, foi determinado através da técnica Potenciométrica (ADAD, 1982).

- cor aparente e cor real, foram medidas de acordo com o método Espectrofotométrico, utilizandose um Espectrofotômetro Micronal B295 em comprimento de onda de $530 \mathrm{~nm}$. Os resultados foram expressos em mg Pt-Co/L. A cor real foi medida após centrifugação por 30 minutos a $300 \mathrm{rpm}$ (APHA AWWA, 1985)

- demanda química de oxigênio (DQO), foi realizada segundo método Reduzido, utilizando-se o aparelho Polilab série 043, digital (POLILAB, 199-).

- cloretos, foi determinado seguindo o método Argentimétrico indireto de Volhard (BACAN et al., 1985).

\section{RESULTADOS E DISCUSSÃO}

\section{Primeira seqüência de testes}

Os valores de $\mathrm{pH}$, verificadas no efluente não filtrado e filtrado, submetidos aos tratamentos com sulfato de alumínio e cloreto férrico são apresentados na Tabela 1. 
TABELA 1: Médias dos valores de $\mathrm{pH}$, nas amostras submetidas aos tratamentos com $\left[\mathrm{Al}\left(\mathrm{SO}_{4}\right)_{3} .14\right.$ $\left.\mathrm{H}_{2} \mathrm{O}\right]$ e $\left[\mathrm{Fe} \mathrm{Cl}_{3} .6 \mathrm{H}_{2} \mathrm{O}\right.$ ], com o tempo de agitação de 1 minuto com alta velocidade e 15 minutos à baixa velocidade.

\begin{tabular}{|c|c|c|c|c|c|c|}
\hline \multirow{2}{*}{$\begin{array}{c}\text { Concentrações } \\
\text { (ppm) }\end{array}$} & \multicolumn{3}{|c|}{ Não filtrado } & \multicolumn{3}{|c|}{ Filtrado } \\
\hline & $\begin{array}{c}\text { Efluente } \\
\text { bruto }\end{array}$ & $\begin{array}{l}{\left[\mathrm{Al}\left(\mathrm{SO}_{4}\right)_{3}\right.} \\
\left..14 \mathrm{H}_{2} \mathrm{O}\right] \\
\end{array}$ & $\begin{array}{l}{\left[\mathrm{FeCl}_{3}\right.} \\
\left.6 \mathrm{H}_{2} \mathrm{O}\right] \\
\end{array}$ & $\begin{array}{c}\text { Efluente } \\
\text { bruto }\end{array}$ & $\begin{array}{l}{\left[\mathrm{Al}\left(\mathrm{SO}_{4}\right)_{3}\right.} \\
\left..14 \mathrm{H}_{2} \mathrm{O}\right] \\
\end{array}$ & $\begin{array}{l}{\left[\mathrm{FeCl}_{3}\right.} \\
\left.6 \mathrm{H}_{2} \mathrm{O}\right] \\
\end{array}$ \\
\hline 0 & $6,13^{a}$ & & & $5,88^{b}$ & & \\
\hline 2500 & & $4,50^{\mathrm{e}}$ & & & $4,53^{\mathrm{e}}$ & \\
\hline 2800 & & $4,83^{\mathrm{c}}$ & & & $4,86^{\mathrm{c}}$ & \\
\hline 3000 & & $4,94^{\mathrm{c}}$ & $4,42^{\text {ef }}$ & & $4,98^{c}$ & $4,56^{\text {de }}$ \\
\hline 3500 & & & $4,12^{\mathrm{g}}$ & & & $4,26^{\mathrm{fg}}$ \\
\hline 4000 & & & $3,50^{\mathrm{h}}$ & & & $3,67^{\mathrm{h}}$ \\
\hline
\end{tabular}

Onde: $\mathrm{a}, \mathrm{b}, \mathrm{c}, \mathrm{d}, \mathrm{e}, \mathrm{f}, \mathrm{g}, \mathrm{h}$, na linha e coluna as médias seguidas por letras diferem significativamente pelo teste $\mathrm{t}$ (PDIFF) ao nível de $\mathrm{P}<0,01$.

Pela Tabela 1 e Figura 1, é possível observar que a filtração do efluente diminuiu ligeiramente o $\mathrm{pH}$, indicando remoção de fragmentos de fibras e macro-moléculas de lignina mais alcalinas. $\mathrm{O}$ aumento na concentração de sulfato de alumínio provoca um aumento linear nos valores de $\mathrm{pH}$, sendo este comportamento semelhante para efluente não filtrado e filtrado. A diminuição da concentração de cloreto férrico resulta no aumento dos valores de $\mathrm{pH}$ tanto, no efluente não filtrado como no filtrado. Valores de pH próximos a 6,0 até 8,5 caracterizam as melhores tratamentos (SECRETARIA DA SAÚDE E DO MEIO AMBIENTE DO RIO GRANDE

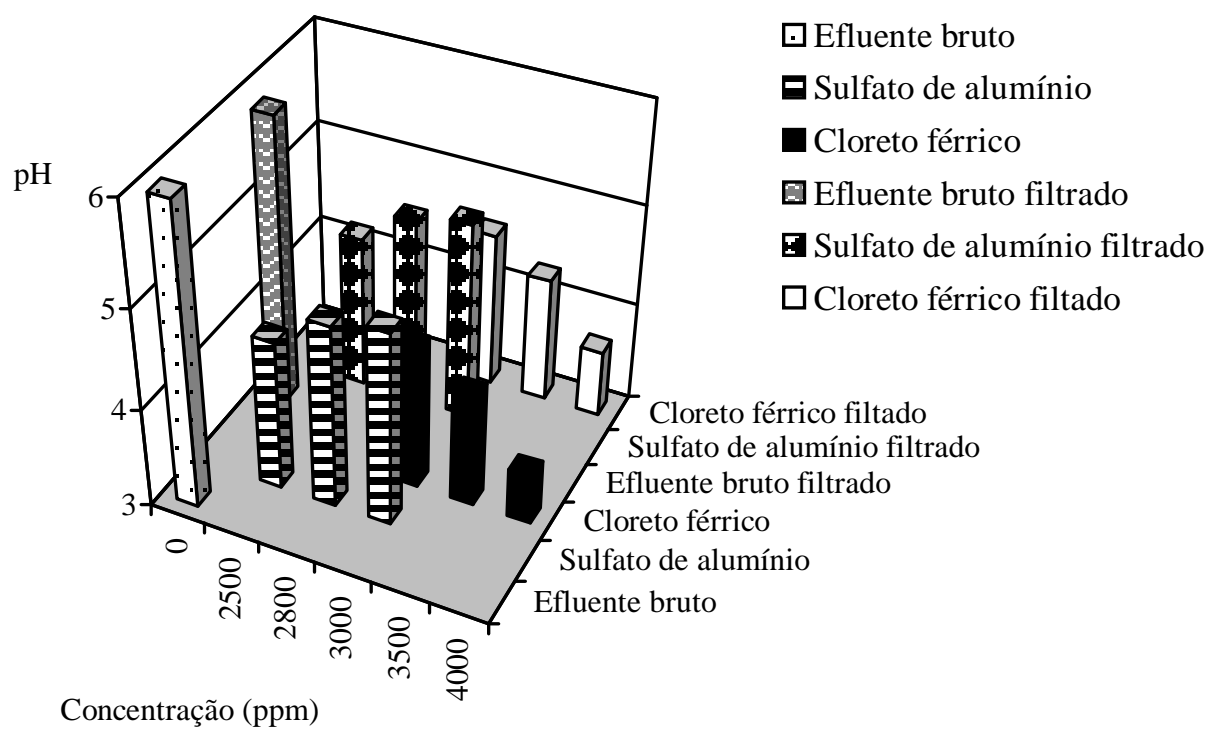

FIGURA 1: Caracterização dos valores de $\mathrm{pH}$ do efluente bruto não filtrado e filtrado submetidos aos tratamentos com sulfato de alumínio e cloreto férrico, não filtrado e filtrado. 
DO SUL, 1989), demostrando que para $\mathrm{pH}$ os tratamentos ficaram abaixo da faixa recomendada.

De acordo com a Tabela 2 a cor aparente foi substancialmente reduzida pela filtração do efluente neutralizado. Concentrações acima de 2.500 ppm de sulfato de alumínio mostraram-se deficientes, pois a cor aumentou à medida em que as concentrações variavam, tanto para o efluente filtrado e não filtrado. Já para cloreto férrico quando o efluente não foi filtrado, os valores de cor aparente aumentaram com o aumento da concentração e para o efluente filtrado os valores diminuíram com o aumento da concentração. Baixos valores de unidades de cor (mg Pt-Co/L), caracterizam os melhores tratamentos (KATO,1983).

TABELA 2: Médias dos valores de cor aparente (mg Pt-Co/L), nas amostras submetidas aos tratamentos com $\left[\mathrm{Al}\left(\mathrm{SO}_{4}\right)_{3} .14 \mathrm{H}_{2} \mathrm{O}\right] \mathrm{e}\left[\mathrm{FeCl}_{3}\right.$. $\left.6 \mathrm{H}_{2} \mathrm{O}\right]$, com o tempo de agitação de 1 minuto com alta velocidade e 15 minutos à baixa velocidade.

\begin{tabular}{|c|c|c|c|c|c|c|}
\hline \multirow{2}{*}{$\begin{array}{c}\text { Concentração } \\
(\mathrm{ppm})\end{array}$} & \multicolumn{3}{|c|}{ Não filtrado } & \multicolumn{3}{|c|}{ Filtrado } \\
\hline & $\begin{array}{c}\text { Efluente } \\
\text { bruto }\end{array}$ & $\begin{array}{l}{\left[\mathrm{Al}\left(\mathrm{SO}_{4}\right)_{3}\right.} \\
\left..14 \mathrm{H}_{2} \mathrm{O}\right]\end{array}$ & $\begin{array}{c}{\left[\mathrm{Fe}(\mathrm{Cl})_{3}\right.} \\
\left..6 \mathrm{H}_{2} \mathrm{O}\right]\end{array}$ & $\begin{array}{c}\text { Efluente } \\
\text { bruto }\end{array}$ & $\begin{array}{l}{\left[\mathrm{Al}\left(\mathrm{SO}_{4}\right)_{3}\right.} \\
\left..14 \mathrm{H}_{2} \mathrm{O}\right]\end{array}$ & $\begin{array}{r}{\left[\mathrm{Fe}(\mathrm{Cl})_{3}\right.} \\
\left..6 \mathrm{H}_{2} \mathrm{O}\right]\end{array}$ \\
\hline 0 & $1992,02^{h}$ & & & $714,74^{\mathrm{g}}$ & & \\
\hline 2500 & & $50,92^{\mathrm{a}}$ & & & $39,72^{\mathrm{a}}$ & \\
\hline 2800 & & $490,90^{d}$ & & & $342,23^{c}$ & \\
\hline 3000 & & $211,77^{b}$ & $859,89^{f}$ & & $246,63^{b}$ & 324,01 bc \\
\hline 3500 & & & $1489,36^{\mathrm{g}}$ & & & $52,46^{\mathrm{a}}$ \\
\hline 4000 & & & $1529,80^{\mathrm{g}}$ & & & $52,46^{\mathrm{a}}$ \\
\hline
\end{tabular}

Onde: $a, b, c, d, e, f, g, h$, na linha e coluna as médias seguidas por letras diferem significativamente pelo teste $\mathrm{t}$ (PDIFF) ao nível de $\mathrm{P}<0,01$.

O comportamento dos valores para cor aparente são apresentados na Figura 2.

$\mathrm{Na}$ Tabela 3 e Figura 3, todos os tratamentos foram igualmente efetivos, reduzindo a cor real para níveis muito baixos. Segundo KATO (1983) baixos valores indicam os melhores tratamentos. Os resultados das médias obtidas para cor real, não apresentaram diferenças significativas com relação aos tratamentos com $\left[\mathrm{Al}\left(\mathrm{SO}_{4}\right)_{3} .14 \mathrm{H}_{2} \mathrm{O}\right]$ e $\left[\mathrm{FeCl}_{3} .6 \mathrm{H}_{2} \mathrm{O}\right]$ filtrado e não filtrado, em todas as concentrações. Na verdade, o que precisa-se melhorar na floculação é a velocidade e qualidade da decantação dos flocos para uma máxima redução de cor. Outra alternativa seria a instalação de filtros de areia ou selite para o efluente sobrenadante da etapa de clarifloculação.

Os dados da Tabela 4 e Figura 4 mostram o efeito da filtração na redução de DQO (praticamente 50\%), entretanto, esta redução pela floculação, nestas concentrações não foi tão expressiva, ficando os melhores resultados por volta de $55 \%$ de redução. As menores médias foram obtidas com a concentração de $2500 \mathrm{ppm}$ de $\left[\mathrm{Al}\left(\mathrm{SO}_{4}\right)_{3}\right.$. $14 \mathrm{H}_{2} \mathrm{O}$ ] filtrado, com um valor de 378,73 $\mathrm{mg} / \mathrm{L}$, mas não apresentou diferença significativa, com relação a $3.000 \mathrm{ppm}\left[\mathrm{FeCl}_{3}\right.$. $\left.6 \mathrm{H}_{2} \mathrm{O}\right]$ filtrado. As médias não atingiram os valores recomendados, que são inferiores a $180 \mathrm{mg} / \mathrm{L}$ caracterizando os melhores tratamentos (SECRETARIA DA SAÚDE E DO MEIO AMBIENTE DO ESTADO DO RIO GRANDE DO SUL, 1989). Esses dados indicam que só um tratamento físico-químico, sem tratamento biológico (aeróbico e/ou anaeróbico), não é tão efetivo na redução de DQO, necessitando grande esforço de otimização e controle, o que foi buscado na segunda 
seqüência de testes. Os resultados indicam ainda que as concentrações utilizadas podem ter sido em excesso.

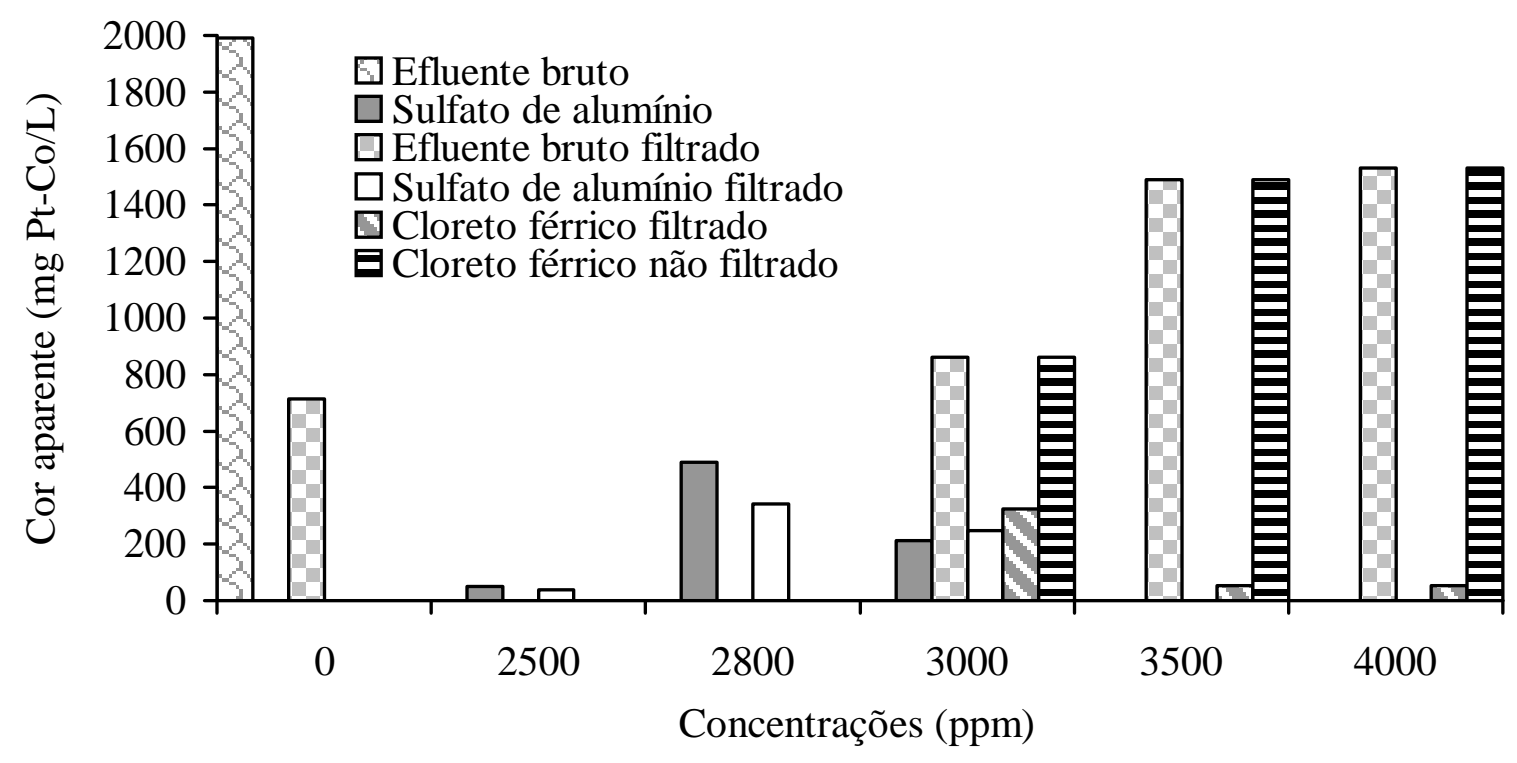

FIGURA 2: Caracterização dos valores de cor aparente em mg Pt-Co/L do efluente bruto não filtrado e filtrado, submetidos aos tratamento com sulfato de alumínio e cloreto férrico ambos não filtrado e filtrado.

TABELA 3: Médias dos valores de cor real (mg Pt-Co/L), nas amostras submetidas aos tratamentos com $\left[\mathrm{Al}\left(\mathrm{SO}_{4}\right)_{3} .14 \mathrm{H}_{2} \mathrm{O}\right]$ e $\left[\mathrm{FeCl}_{3} .6 \mathrm{H}_{2} \mathrm{O}\right]$, com o tempo de agitação de 1 minuto com alta velocidade e 15 minutos à baixa velocidade.

\begin{tabular}{|c|c|c|c|c|c|c|}
\hline \multirow{2}{*}{$\begin{array}{l}\text { Concentração } \\
\text { (ppm) }\end{array}$} & \multicolumn{3}{|c|}{ Não filtrado } & \multicolumn{3}{|c|}{ Filtrado } \\
\hline & $\begin{array}{c}\text { Efluente } \\
\text { bruto }\end{array}$ & $\begin{array}{r}{\left[\mathrm{Al}\left(\mathrm{SO}_{4}\right)_{3}\right.} \\
\left..14 \mathrm{H}_{2} \mathrm{O}\right]\end{array}$ & $\begin{array}{l}{\left[\mathrm{FeCl}_{3}\right.} \\
\left.6 \mathrm{H}_{2} \mathrm{O}\right]\end{array}$ & $\begin{array}{c}\text { Efluente } \\
\text { bruto }\end{array}$ & $\begin{array}{l}{\left[\mathrm{Al}\left(\mathrm{SO}_{4}\right)_{3}\right.} \\
\left..14 \mathrm{H}_{2} \mathrm{O}\right]\end{array}$ & $\begin{array}{l}{\left[\mathrm{FeCl}_{3}\right.} \\
\left.6 \mathrm{H}_{2} \mathrm{O}\right]\end{array}$ \\
\hline 0 & $1130,50^{c}$ & & & $690,47^{b}$ & & \\
\hline 2500 & & $30,32^{\mathrm{a}}$ & & & $27,89^{\mathrm{a}}$ & \\
\hline 2800 & & $27,89^{\mathrm{a}}$ & & & $27,89^{\mathrm{a}}$ & \\
\hline 3000 & & $27,89^{\mathrm{a}}$ & $27,89^{\mathrm{a}}$ & & $27,89^{\mathrm{a}}$ & $27,89^{\mathrm{a}}$ \\
\hline
\end{tabular}

Onde: $\mathrm{a}, \mathrm{b}, \mathrm{c}$, na linha e coluna as médias seguidas por letras diferem significativamente pelo teste $\mathrm{t}$ (PDIFF) ao nível de $\mathrm{P}<0,01$. 


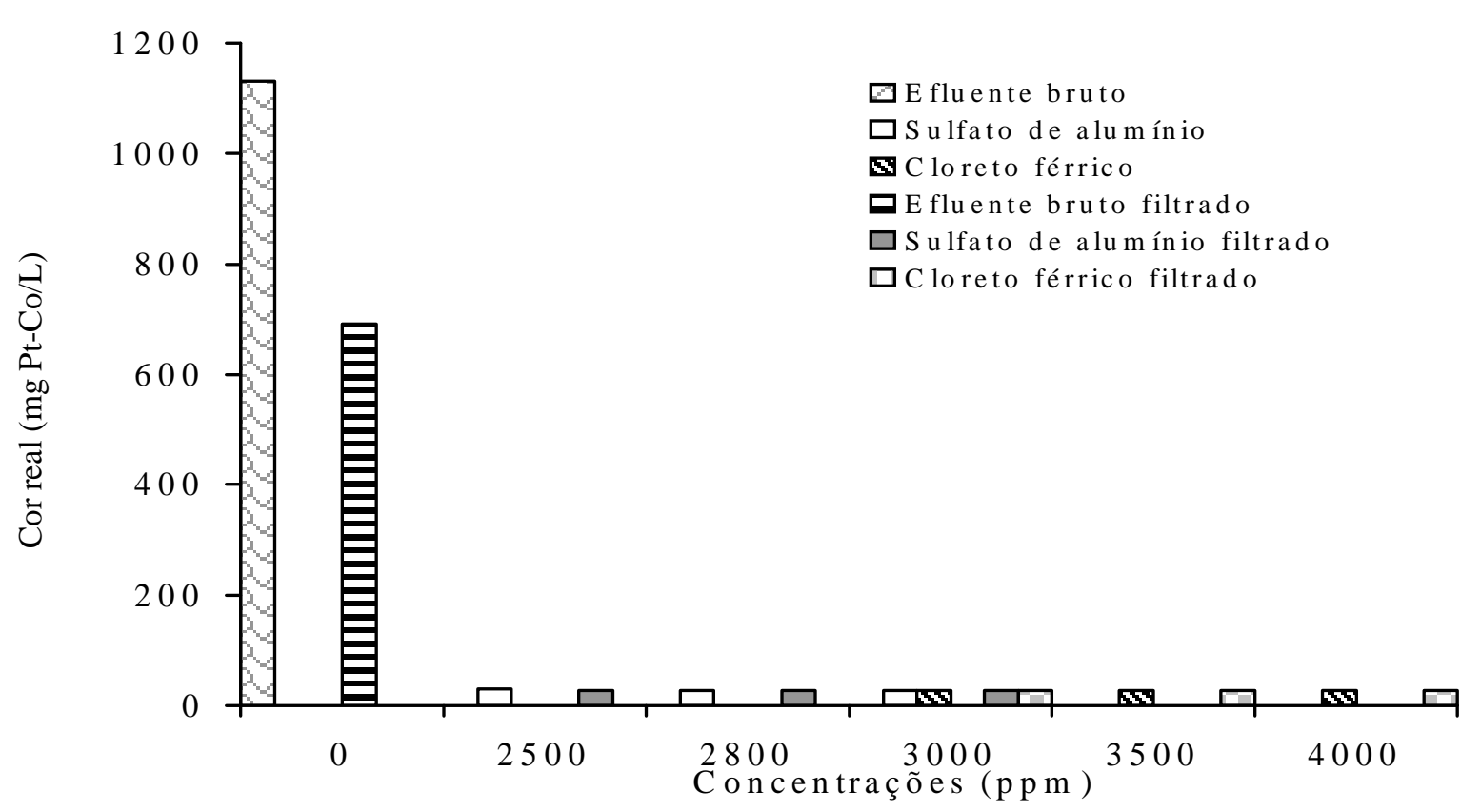

FIGURA 3: Caracterização dos valores de cor real em mg Pt-Co/L do efluente bruto não filtrado e filtrado, submetidos aos tratamento com sulfato de alumínio e cloreto férrico ambos não filtrado e filtrado.

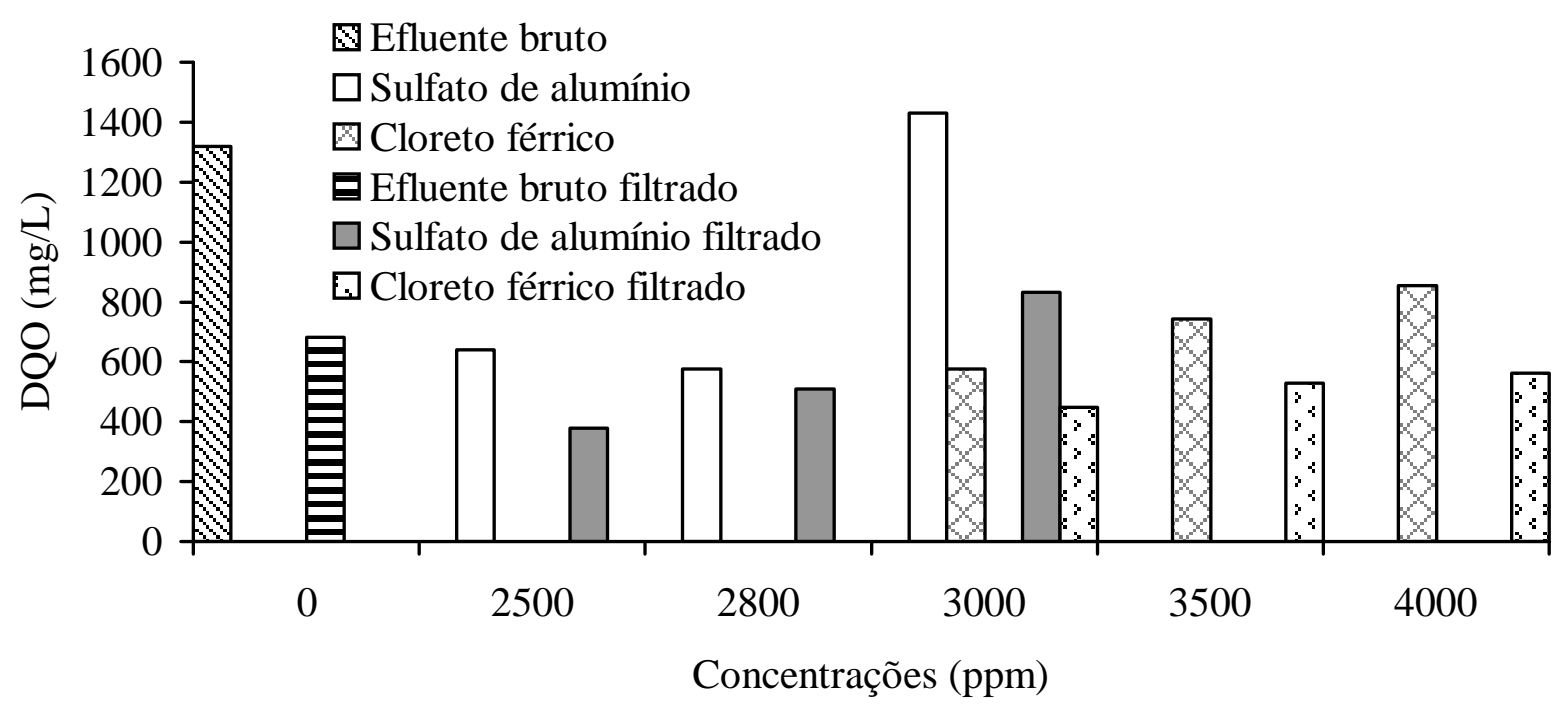

FIGURA 4: Caracterização dos valores de DQO (mg/L) do efluente bruto não filtrado e filtrado, submetidos aos tratamento com sulfato de alumínio e cloreto férrico ambos não filtrado e filtrado. 
TABELA 4: Médias dos valores de DQO (mg/L), nas amostras submetidas aos tratamentos com $\left[\mathrm{Al}\left(\mathrm{SO}_{4}\right)_{3} .14 \mathrm{H}_{2} \mathrm{O}\right]$ e $\left[\mathrm{FeCl}_{3} \cdot 6 \mathrm{H}_{2} \mathrm{O}\right]$, com o tempo de agitação de 1 minuto com alta velocidade e 15 minutos à baixa velocidade.

\begin{tabular}{|c|c|c|c|c|c|c|}
\hline \multirow{2}{*}{$\begin{array}{c}\text { Concentração } \\
(\mathrm{ppm})\end{array}$} & \multicolumn{3}{|c|}{ Não filtrado } & \multicolumn{3}{|c|}{ Filtrado } \\
\hline & $\begin{array}{c}\text { Efluente } \\
\text { bruto }\end{array}$ & $\begin{array}{l}{\left[\mathrm{Al}\left(\mathrm{SO}_{4}\right)_{3}\right.} \\
\left..14 \mathrm{H}_{2} \mathrm{O}\right]\end{array}$ & $\begin{array}{l}{\left[\mathrm{FeCl}_{3}\right.} \\
\left.6 \mathrm{H}_{2} \mathrm{O}\right]\end{array}$ & $\begin{array}{c}\text { Efluente } \\
\text { Bruto }\end{array}$ & $\begin{array}{r}{\left[\mathrm{Al}\left(\mathrm{SO}_{4}\right)_{3}\right.} \\
\left.12 \mathrm{H}_{2} \mathrm{O}\right]\end{array}$ & $\begin{array}{l}\mathrm{FeCl}_{3} \\
6 \mathrm{H}_{2} \mathrm{O}\end{array}$ \\
\hline 0 & $1317,78^{f}$ & & & $682,22^{\mathrm{de}}$ & & \\
\hline 2500 & & $640,93^{c}$ & & & $378,73^{a}$ & \\
\hline 2800 & & $576,62^{b c}$ & & & $508,56^{b}$ & \\
\hline 3000 & & $1429,91^{\mathrm{g}}$ & $576,47^{b c}$ & & $831,91^{\text {de }}$ & $449,31^{\mathrm{ab}}$ \\
\hline 3500 & & & $742,47^{\mathrm{d}}$ & & & $529,19^{b}$ \\
\hline 4000 & & & $853,31^{d}$ & & & $563,42^{\text {bc }}$ \\
\hline
\end{tabular}

Onde: $\mathrm{a}, \mathrm{b}, \mathrm{c}, \mathrm{d}, \mathrm{e}, \mathrm{f}, \mathrm{g}$, na linha e coluna as médias seguidas por letras diferem significativamente pelo teste $\mathrm{t}$ (PDIFF) ao nível de $\mathrm{P}<0,01$.

A Figura 5 e a Tabela 5 ilustram o comportamento dos cloretos em relação as diferentes concentrações de $\left[\mathrm{Al}\left(\mathrm{SO}_{4}\right)_{3} .14 \mathrm{H}_{2} \mathrm{O}\right]$ e $\left[\mathrm{FeCl}_{3}\right.$. $\left.6 \mathrm{H}_{2} \mathrm{O}\right]$.

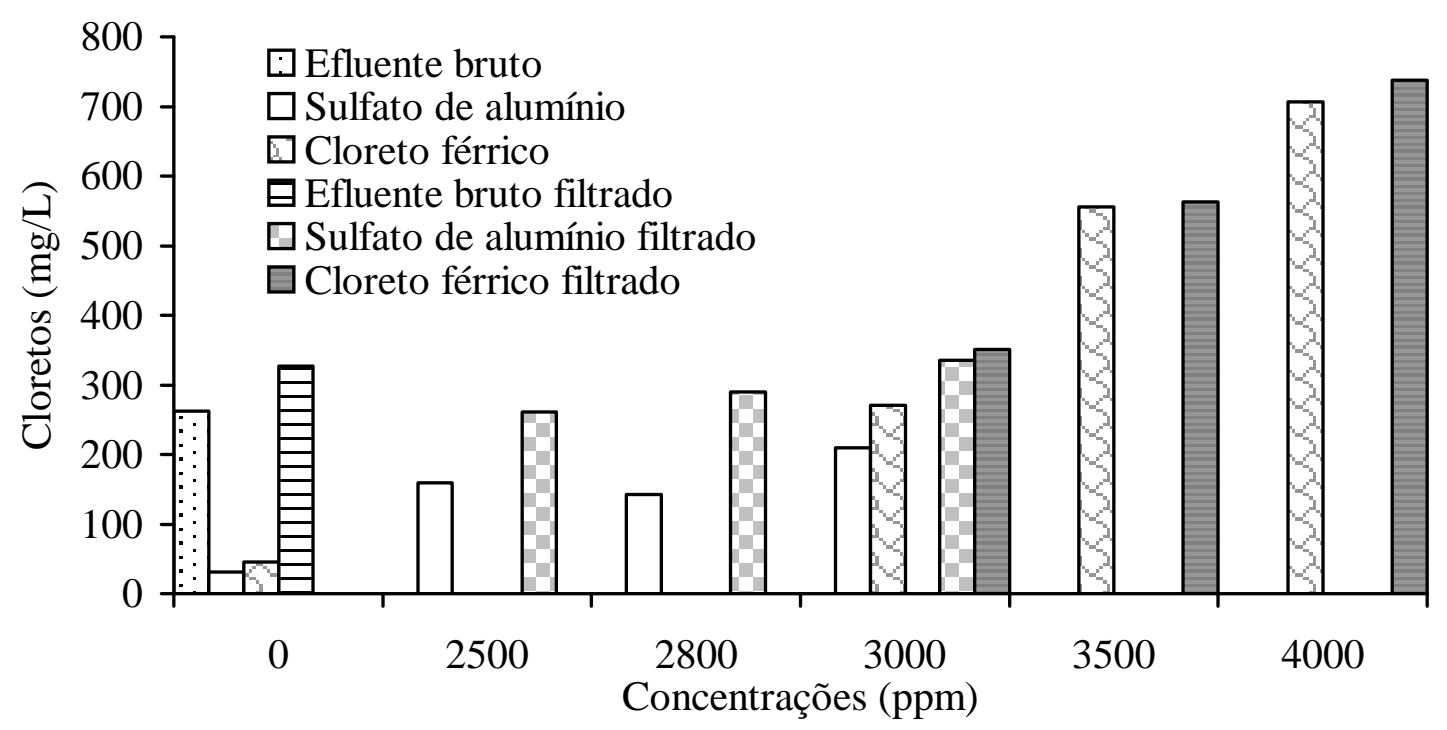

FIGURA 5: Caracterização dos valores de cloretos $(\mathrm{mg} / \mathrm{L})$ do efluente bruto não filtrado e filtrado, submetidos aos tratamento com sulfato de alumínio e cloreto férrico ambos não filtrado e filtrado. 
TABELA 5: Médias dos valores de cloretos (mg/L), nas amostras submetidas aos tratamentos com $\left[\mathrm{Al}\left(\mathrm{SO}_{4}\right)_{3} .14 \mathrm{H}_{2} \mathrm{O}\right]$ e $\left[\mathrm{FeCl}_{3} \cdot 6 \mathrm{H}_{2} \mathrm{O}\right]$, com o tempo de agitação de 1 minuto com alta velocidade e 15 minutos à baixa velocidade.

\begin{tabular}{|c|c|c|c|c|c|c|}
\hline \multirow{2}{*}{$\begin{array}{l}\text { Concentração } \\
\text { (ppm) }\end{array}$} & \multicolumn{3}{|c|}{ Não filtrado } & \multicolumn{3}{|c|}{ Filtrado } \\
\hline & $\begin{array}{c}\text { Efluente } \\
\text { bruto }\end{array}$ & $\begin{array}{l}{\left[\mathrm{Al}\left(\mathrm{SO}_{4}\right)_{3}\right.} \\
\left..14 \mathrm{H}_{2} \mathrm{O}\right]\end{array}$ & $\begin{array}{l}{\left[\mathrm{FeCl}_{3}\right.} \\
\left.6 \mathrm{H}_{2} \mathrm{O}\right]\end{array}$ & $\begin{array}{c}\text { Efluente } \\
\text { bruto }\end{array}$ & $\begin{array}{l}{\left[\mathrm{Al}\left(\mathrm{SO}_{4}\right)_{3}\right.} \\
\left..14 \mathrm{H}_{2} \mathrm{O}\right]\end{array}$ & $\begin{array}{l}{\left[\mathrm{FeCl}_{3}\right.} \\
\left.6 \mathrm{H}_{2} \mathrm{O}\right]\end{array}$ \\
\hline 0 & $262,10^{c}$ & & & $327,12^{d}$ & & \\
\hline 2500 & & $159,29^{\mathrm{a}}$ & & & $261,40^{\mathrm{c}}$ & \\
\hline 2800 & & $142,25^{\mathrm{a}}$ & & & $290,22^{c}$ & \\
\hline 3000 & & $209,49^{b}$ & $271,12^{\mathrm{c}}$ & & $335,58^{d}$ & $351,45^{d}$ \\
\hline 3500 & & & $555,89^{\mathrm{e}}$ & & & $562,76^{\mathrm{e}}$ \\
\hline 4000 & & & $706,58^{f}$ & & & $737,46^{\mathrm{g}}$ \\
\hline
\end{tabular}

Onde: $\mathrm{a}, \mathrm{b}, \mathrm{c}, \mathrm{d}, \mathrm{e}, \mathrm{f}, \mathrm{g}$, na linha e coluna as médias seguidas por letras diferem significativamente pelo teste $\mathrm{t}$ (PDIFF) ao nível de $\mathrm{P}<0,01$.

Como era de se esperar, de acordo com FRIZZO (1993) a Tabela 5 e Figura 5 e mostra que as concentrações crescentes de cloreto férrico aumentam o teor de cloretos. Como esse índice é controlado pela legislação $(500 \mathrm{mg} / \mathrm{L})$, o uso de cloreto férrico fica prejudicado até certo ponto, exigindo maiores cuidados.

Por outro lado, a floculação com sulfato de alumínio colabora para a redução do teor de cloretos do efluente (até 40-45\%). Isso deve estar relacionado ao arraste de moléculas orgânicas com íons cloretos trocáveis, quando da floculação. A Figura 5 ilustra o comportamento do efluente.

Devido ao fato de que o $\left[\mathrm{Al}_{2}\left(\mathrm{SO}_{4}\right)_{3} \cdot 14 \mathrm{H}_{2} \mathrm{O}\right]$ (na concentração de $2500 \mathrm{ppm}$ ) na primeira seqüência de testes ter sido o melhor tratamento para a redução de cloretos, e para aumentar o pH (2800 ppm), a fim de deixá-lo dentro ou próximo aos valores legislados, buscou-se utilizar concentrações menores para minimizar os custos e o tempo.

\section{Segunda seqüência de testes}

Como se pode verificar com a Tabela 6 , esta nova amostra de efluente possuía um pH ligeiramente superior. Após os tratamentos os valores foram diminuídos, sendo o $\mathrm{pH}(5,44)$, com o efluente filtrado, com a concentração de $1.000 \mathrm{ppm}$ de $\left[\mathrm{Al}_{2}\left(\mathrm{SO}_{4}\right)_{3} \cdot 14 \mathrm{H}_{2} \mathrm{O}\right]$ o tratamento que mais se aproximou da faixa legislada $(6,0-8,5)$.

Os resultados da Tabela 7 indicam como melhores, as concentrações próximas a 1.000 ppm para máxima redução da cor aparente. Para o efluente filtrado, todas as concentrações mostram bons resultados. O tempo de 15 minutos de agitação lenta mostrou-se mais indicado, (46,39 mg Pt-Co/L), em virtude de não apresentar diferenças significativas em relação aos outros tratamentos. 
TABELA 6: Médias dos valores de $\mathrm{pH}$ nas amostras submetidas ao tratamento com $\left[\mathrm{Al}\left(\mathrm{SO}_{4}\right)_{3}\right.$. 14 $\left.\mathrm{H}_{2} \mathrm{O}\right]$, com tempos de agitações de 1 minuto com alta velocidade e 15 e 10 minutos à baixa velocidade.

\begin{tabular}{|c|c|c|c|c|c|c|}
\hline \multirow{4}{*}{$\begin{array}{l}\text { Concentração } \\
\text { (ppm) }\end{array}$} & \multicolumn{3}{|c|}{ Não filtrado } & \multicolumn{3}{|c|}{ Filtrado } \\
\hline & $\begin{array}{c}\text { Efluente } \\
\text { bruto }\end{array}$ & \multicolumn{2}{|c|}{$\left[\mathrm{Al}\left(\mathrm{SO}_{4}\right)_{3} .14 \mathrm{H}_{2} \mathrm{O}\right]$} & $\begin{array}{c}\text { Efluente } \\
\text { bruto }\end{array}$ & \multicolumn{2}{|c|}{$\left[\mathrm{Al}\left(\mathrm{SO}_{4}\right)_{3} .14 \mathrm{H}_{2} \mathrm{O}\right]$} \\
\hline & \multicolumn{3}{|c|}{ Tempo de agitação } & \multicolumn{3}{|c|}{ Tempo de agitação } \\
\hline & 0 & $10 \mathrm{~min}$. & $15 \mathrm{~min}$. & 0 & $10 \mathrm{~min}$. & $15 \mathrm{~min}$. \\
\hline 0 & $7,6^{\mathrm{b}}$ & & & $7,83^{a}$ & & \\
\hline 1000 & & $5,27^{\mathrm{cd}}$ & $5,24^{\mathrm{d}}$ & & $5,44^{\mathrm{c}}$ & $5,30^{\mathrm{cd}}$ \\
\hline 1500 & & $5,33^{c}$ & $5,10^{d}$ & & $5,10^{d}$ & $5,19^{d}$ \\
\hline 2000 & & $5,17^{\mathrm{d}}$ & $5,21^{\mathrm{d}}$ & & $5,29^{\mathrm{cd}}$ & $5,16^{\mathrm{d}}$ \\
\hline
\end{tabular}

Onde: $a, b, c, d$, na linha e coluna as médias seguidas por letras diferem significativamente pelo teste $\mathrm{t}$ (PDIFF) ao nível de $\mathrm{P}<0,01$.

TABELA 7: Médias dos valores obtidos de cor aparente (mg Pt-Co/L) nas amostras submetidas ao tratamento com $\left[\mathrm{Al}\left(\mathrm{SO}_{4}\right)_{3} .14 \mathrm{H}_{2} \mathrm{O}\right]$, com tempos de agitações de 1 minuto com alta velocidade e 15 e 10 minutos à baixa velocidade.

\begin{tabular}{|c|c|c|c|c|c|c|}
\hline \multirow{4}{*}{$\begin{array}{l}\text { Concentração } \\
\text { (ppm) }\end{array}$} & \multicolumn{3}{|c|}{ Não filtrado } & \multicolumn{3}{|c|}{ Filtrado } \\
\hline & $\begin{array}{c}\text { Efluente } \\
\text { bruto }\end{array}$ & \multicolumn{2}{|c|}{$\left[\mathrm{Al}\left(\mathrm{SO}_{4}\right)_{3} .14 \mathrm{H}_{2} \mathrm{O}\right]$} & $\begin{array}{c}\text { Efluente } \\
\text { bruto }\end{array}$ & \multicolumn{2}{|c|}{$\left[\mathrm{Al}\left(\mathrm{SO}_{4}\right)_{3} .14 \mathrm{H}_{2} \mathrm{O}\right]$} \\
\hline & \multicolumn{3}{|c|}{ Tempo de agitação } & \multicolumn{3}{|c|}{ Tempo de agitação } \\
\hline & 0 & $10 \mathrm{~min}$. & $15 \mathrm{~min}$. & 0 & $10 \mathrm{~min}$. & $15 \mathrm{~min}$. \\
\hline 0 & $2926,47^{\mathrm{e}}$ & & & $1949,55^{c}$ & & \\
\hline 1000 & & $117,73^{\mathrm{d}}$ & $349,79^{c}$ & & $55,50^{\mathrm{ab}}$ & $46,39^{a b}$ \\
\hline 1500 & & $99,49^{\mathrm{ab}}$ & $64,60^{a}$ & & $42,15^{\mathrm{a}}$ & $32,74^{\mathrm{a}}$ \\
\hline 2000 & & $57,01^{\mathrm{a}}$ & $57,01^{\mathrm{a}}$ & & $41,84^{\mathrm{a}}$ & $33,96^{\mathrm{a}}$ \\
\hline
\end{tabular}

Onde: $a, b, c, d, e$, na linha e coluna as médias seguidas por letras diferem significativamente pelo teste $\mathrm{t}$ (PDIFF) ao nível de $\mathrm{P}<0,01$.

De acordo com a Tabela 8 , da mesma forma que na primeira seqüência de testes inicial, a cor real foi significativamente reduzida em todos os casos, não havendo diferenças significativas, a melhor concentração foi de 1.000 ppm de $\left[\mathrm{Al}_{2}\left(\mathrm{SO}_{4}\right)_{3} .14 \mathrm{H}_{2} \mathrm{O}\right]$ à baixa velocidade com dez minutos. Isto reforça a necessidade de se melhorar a decantação para remoção dos microflocos que se mantém suspensos no efluente.

Conforme a Tabela 9, a redução da concentração de sulfato de alumínio permitiu alcançar reduções de $80 \%$ da DQO, o que pode ser considerado excelente para o tratamento em etapa única. Também para DQO a agitação lenta por mais tempo (15 minutos) foi benéfica (306,22 mg/L), com a concentração de $1.500 \mathrm{ppm}$ de $\left[\mathrm{Al}_{2}\left(\mathrm{SO}_{4}\right)_{3} \cdot 14 \mathrm{H}_{2} \mathrm{O}\right]$, filtrado, em virtude deste tratamento não apresentar diferenças significativas em relação aos demais. 
TABELA 8: Médias dos valores de cor real (mg Pt-Co/L) nas amostras submetidas ao tratamento com $\left[\mathrm{Al}\left(\mathrm{SO}_{4}\right)_{3} .14 \mathrm{H}_{2} \mathrm{O}\right]$, com tempos de agitações de 1 minuto com alta velocidade e 15 e 10 minutos à baixa velocidade.

\begin{tabular}{|c|c|c|c|c|c|c|}
\hline \multirow{4}{*}{$\begin{array}{c}\text { Concentração } \\
(\mathrm{ppm})\end{array}$} & \multicolumn{3}{|c|}{ Não filtrado } & \multicolumn{3}{|c|}{ Filtrado } \\
\hline & $\begin{array}{c}\text { Efluente } \\
\text { bruto }\end{array}$ & \multicolumn{2}{|c|}{$\left[\mathrm{Al}\left(\mathrm{SO}_{4}\right)_{3} \cdot 14 \mathrm{H}_{2} \mathrm{O}\right]$} & $\begin{array}{c}\text { Efluente } \\
\text { bruto }\end{array}$ & \multicolumn{2}{|c|}{$\left[\mathrm{Al}\left(\mathrm{SO}_{4}\right)_{3} \cdot 14 \mathrm{H}_{2} \mathrm{O}\right]$} \\
\hline & \multicolumn{3}{|c|}{ Tempo de agitação } & \multicolumn{3}{|c|}{ Tempo de agitação } \\
\hline & 0 & $10 \mathrm{~min}$. & $15 \mathrm{~min}$. & 0 & $10 \mathrm{~min}$. & $15 \mathrm{~min}$. \\
\hline 0 & 1834,26 & & & $680,48^{\mathrm{b}}$ & & \\
\hline 1000 & & $35,07^{\mathrm{a}}$ & $27,89^{\mathrm{a}}$ & & $27,89^{\mathrm{a}}$ & $27,89^{\mathrm{a}}$ \\
\hline 1500 & & $27,89^{\mathrm{a}}$ & $27,89^{\mathrm{a}}$ & & $27,89^{\mathrm{a}}$ & $27,89^{\mathrm{a}}$ \\
\hline 2000 & & $27,89^{\mathrm{a}}$ & $27,89^{\mathrm{a}}$ & & $27,89^{\mathrm{a}}$ & $27,89^{\mathrm{a}}$ \\
\hline
\end{tabular}

Onde: $\mathrm{a}, \mathrm{b}, \mathrm{c}$, na linha e coluna as médias seguidas por letras diferem significativamente pelo teste $\mathrm{t}$ (PDIFF) ao nível de $\mathrm{P}<0,01$.

TABELA 9: Médias dos valores de DQO (mg/L) nas amostras submetidas ao tratamento com $\left[\mathrm{Al}\left(\mathrm{SO}_{4}\right)_{3} .14 \mathrm{H}_{2} \mathrm{O}\right]$, com tempos de agitações de 1 minuto com alta velocidade e $15 \mathrm{e}$ 10 minutos à baixa velocidade.

\begin{tabular}{|c|c|c|c|c|c|c|}
\hline \multirow{4}{*}{$\begin{array}{l}\text { Concentração } \\
(\mathrm{ppm})\end{array}$} & \multicolumn{3}{|c|}{ Não filtrado } & \multicolumn{3}{|c|}{ Filtrado } \\
\hline & $\begin{array}{c}\text { Efluente } \\
\text { bruto }\end{array}$ & \multicolumn{2}{|c|}{$\left[\mathrm{Al}\left(\mathrm{SO}_{4}\right)_{3} .14 \mathrm{H}_{2} \mathrm{O}\right]$} & $\begin{array}{c}\text { Efluente } \\
\text { bruto }\end{array}$ & \multicolumn{2}{|c|}{$\left[\mathrm{Al}\left(\mathrm{SO}_{4}\right)_{3} .14 \mathrm{H}_{2} \mathrm{O}\right]$} \\
\hline & \multicolumn{3}{|c|}{ Tempo de agitação } & \multicolumn{3}{|c|}{ Tempo de agitação } \\
\hline & 0 & $10 \mathrm{~min}$. & $15 \mathrm{~min}$. & 0 & $10 \mathrm{~min}$. & $15 \mathrm{~min}$. \\
\hline 0 & $1313,33^{f}$ & & & $767,22^{d}$ & & \\
\hline 1000 & & $1075,20^{\mathrm{e}}$ & $750,22^{d}$ & & $367,18^{b c}$ & $308,29^{b}$ \\
\hline 1500 & & $409,07^{\mathrm{c}}$ & $286,98^{a b}$ & & 351,29 de & $306,22^{a b}$ \\
\hline 2000 & & $259,44^{\mathrm{ab}}$ & $241,27^{\mathrm{ab}}$ & & $222,22^{\mathrm{a}}$ & $203,27^{\mathrm{a}}$ \\
\hline
\end{tabular}

Onde: $a, b, c, d, e, f$, na linha e coluna as médias seguidas por letras diferem significativamente pelo teste $\mathrm{t}$ (PDIFF) ao nível de $\mathrm{P}<0,01$.

Com base nos resultados obtidos na Tabela 10 o efluente dessa seqüência de testes era bem mais rico em cloretos (cerca do dobro). As reduções chegaram a $20 \%$ com a concentração de 2.000 ppm de sulfato de alumínio, 15 minutos de agitação lenta, para o efluente bruto não filtrado. Apesar de não ser expressiva, é uma redução que pode ajudar a enquadrar um efluente no valor legislado (500 mg/L), o melhor valor foi de $(420,74 \mathrm{mg} / \mathrm{L})$. 
TABELA 10: Médias dos valores obtidos de cloretos $(\mathrm{mg} / \mathrm{L})$ nas amostras submetidas ao tratamento com $\left[\mathrm{Al}\left(\mathrm{SO}_{4}\right)_{3} .14 \mathrm{H}_{2} \mathrm{O}\right]$, com tempos de agitações de 1 minuto com alta velocidade e 15 e 10 minutos à baixa velocidade.

\begin{tabular}{|c|c|c|c|c|c|c|}
\hline \multirow{4}{*}{$\begin{array}{c}\text { Concentração } \\
(\mathrm{ppm})\end{array}$} & \multicolumn{3}{|c|}{ Não filtrado } & \multicolumn{3}{|c|}{ Filtrado } \\
\hline & $\begin{array}{c}\text { Efluente } \\
\text { bruto }\end{array}$ & \multicolumn{2}{|c|}{$\left[\mathrm{Al}\left(\mathrm{SO}_{4}\right)_{3} .14 \mathrm{H}_{2} \mathrm{O}\right]$} & $\begin{array}{c}\text { Efluente } \\
\text { bruto }\end{array}$ & \multicolumn{2}{|c|}{$\left[\mathrm{Al}\left(\mathrm{SO}_{4}\right)_{3} .14 \mathrm{H}_{2} \mathrm{O}\right]$} \\
\hline & \multicolumn{3}{|c|}{ Tempo de agitação } & \multicolumn{3}{|c|}{ Tempo de agitação } \\
\hline & 0 & $10 \mathrm{~min}$. & $15 \mathrm{~min}$. & 0 & $10 \mathrm{~min}$. & $15 \mathrm{~min}$. \\
\hline 0 & $540,98^{\mathrm{e}}$ & & & $514,45^{d}$ & & \\
\hline 1000 & & $467,76^{b}$ & $480,38^{b c}$ & & $461,67^{b}$ & $463,74^{b}$ \\
\hline 1500 & & $479,63^{\mathrm{bc}}$ & $507,42^{d}$ & & $484,64^{\mathrm{c}}$ & $513,60^{d}$ \\
\hline 2000 & & $511,08^{d}$ & $420,74^{\mathrm{a}}$ & & $498,73^{\mathrm{cd}}$ & $468,93^{b c}$ \\
\hline
\end{tabular}

Onde: $a, b, c, d, e$, na linha e coluna as médias seguidas por letras diferem significativamente pelo teste $\mathrm{t}$ (PDIFF) ao nível de $\mathrm{P}<0,01$.

\section{CONCLUSÕES}

As principais conclusões obtidas no trabalho foram as seguintes:

- a filtração do efluente bruto neutralizado, removendo fibras, finos, macromoléculas e flocos naturais, permite expressivas reduções da cor aparente, cor real e DQO do mesmo;

- sulfato de alumínio mostrou-se mais efetivo como coagulante /floculante que o cloreto férrico;

- para a redução da cor aparente $(46,39 \mathrm{mg} \mathrm{Pt}-\mathrm{Co} / \mathrm{L})$ o melhor tratamento com $\left[\mathrm{Al}_{2}\left(\mathrm{SO}_{4}\right)_{3}\right.$. 14 $\mathrm{H}_{2} \mathrm{O}$ ] foi de $1.000 \mathrm{ppm}$, filtrado; para DQO $2.000 \mathrm{ppm}(306,22 \mathrm{mg} / \mathrm{L})$ filtrado e para cloretos 2.000 ppm não filtrado, ao tempo de agitação de 15 minutos à baixa velocidade (50 rpm);

- para a redução da variável cor real e para manter o pH próximo a legislação a melhor concentração de $\left[\mathrm{Al}_{2}\left(\mathrm{SO}_{4}\right)_{3} .14 \mathrm{H}_{2} \mathrm{O}\right]$ foi de $1.000 \mathrm{ppm}$, filtrado ao tempo de agitação de 10 minutos à baixa velocidade $(50 \mathrm{rpm})$;

- os excelentes resultados para cor real indicam que deve-se procurar melhorar as etapas de decantação e separação dos micro-flocos do efluente tratado, filtrações com filtro de areia ou selite (diatomita) podem ser uma efetiva maneira de se alcançar um efluente final com excelente qualidade, em duas etapas de tratamento: clarificação/decantação e filtração;

- as indústrias que operam com o mesmo sistema, no caso de pane em seu tratamento de efluentes, poderão vir a aplicar as técnicas propostas neste estudo, como alternativa, uma vez que os melhores tratamentos foram obtidos com as menores concentrações de $\left[\mathrm{Al}_{2}\left(\mathrm{SO}_{4}\right)_{3} \cdot 14 \mathrm{H}_{2} \mathrm{O}\right]$, o que dará economia de insumos e de tempo. 


\section{AGRADECIMENTOS}

À RIOCELL S.A. pelo auxílio financeiro para a realização deste estudo.

Ao Professor José Henrique Souza e Silva do Departamento de Zootecnia da Universidade Federal de Santa Maria, pela colaboração nas análises estatísticas.

\section{REFERÊNCIAS BIBLIOGRÁFICAS}

ADAD, J.M.T. Controle químico de qualidade. Rio de Janeiro: Guanabara Dois, 1982. 204p.

APHA AWWA. Standard methods for the examination of water and wastewater. Washington, 1985. $1268 \mathrm{p}$.

BACAN, N., ANDRADE, J.C., GODINHO, O.E.S. et al. Química analítica quantitativa elementar . Campinas: Edgard Blücher Ltda., 1985, 259p.

BERTOLLETI, E.. Toxicidade e concentração de agentes tóxicos em efluentes industriais. Ciência e Cultura, São Paulo, v.14, n.3/4, p.271-277, 1990.

BORGES, A. M., COUTO, C., FABRES, A. S. Evolução do efluente, produção utilização do lodo biológico na Cenibra. O Papel, São Paulo, n.07, p.36-41,1994.

CINQUE, U. C., QUADROS, R. A., ANDRADE, M. A. D. Avaliação dos efeitos ambientais de acordo com a BS 7750-Uma experiência real. O Papel, São Paulo, n.01, p.29-34, 1996.

DIN. Determination of adsorbable organically bonded halogens (AOX): DIN 38409. Germany, 1985. part 14.

ERIKSSON, K. L. New development for purification of waste bleach waster. In: ENVIRONMENTAL CONFERENCE, 1991, Atlanta. Anais... Atlanta: Tappi Press, 1991. V.1. p.427-431.

FRIZZO, S. M. B. Investigação sobre a floculação/clarifloculação de efluente secundário biológico, utilizando carvão ativo, resíduos sólidos e excedente do processo da indústria de celulose kraft branqueada. Santa Maria: UFSM, 1993. 137p. Dissertação (Mestrado em Engenharia Florestal) - Universidade Federal de Santa Maria, 1993.

FRIZZO, S. M. B., MARTINS, A. F., FOELKEL, C. E. B. et al. Proposição para redução do teor de cloretos e de elementos-traço de efluentes de indústria de celulose kraft branqueada. O Papel, São Paulo, n. 12, p.147-149, 1996.

FROEHLICH, C. Melhoramento de efluentes da indústria de celulose e papel por carvão biológico. Porto Alegre: UFRGS, 1987. 166p. Dissertação (Mestrado em Engenharia) universidade Federal de Santa Maria, 1987.

HOUK, V. S.. The genotoxicity of industrial wastes and effluents- A Review. Mutation Research, v.277, p.91-138, 1992. 
KATO, M. Controle de poluição das águas em indústrias de celulose. Curso Panamericano de Especialização em Celulose e Papel. 1983. 52p. mimiografado.

LANDIM, A. B., OLIVEIRA, M. B. Sistema de monitoramento contínuo de efluentes em uma planta de celulose. In: $28^{\circ}$ Congresso Anual de celulose e papel, São Paulo.São Paulo - SP Anais... São Paulo: 1995. 1002p. p.629-644.

POLILAB: Método para determinação de DQO. São Paulo: [199-]. 6 p. Catálogo.

SECRETARIA DA SAÚDE E DO MEIO AMBIENTE DO RIO GRANDE DO SUL. Norma Técnica SSMA n $\square$ 01/89. Diário Oficial do Estado, Porto Alegre, 19 mar. 1989.

SUPERSULFATO INDÚSTRIA QUÍMICA Sulfato de alumínio. [s. 1., 1988]. 22p. (Boletim Técnico). 